\title{
100 anos de insulina: como a descoberta do hormônio revolucionou o tratamento de
}

\section{diabetes tipo 1}

100 years of insulin: how the discovery of the hormone revolutionized the treatment of type 1

diabetes

100 años de insulina: cómo el descubrimiento de la hormona revolucionó el tratamiento de la

diabetes tipo 1

Recebido: 03/11/2021 | Revisado: 11/11/2021 | Aceito: 18/11/2021 | Publicado: 28/11/2021

Luiza Lunardi de Freitas

ORCID: https://orcid.org/0000-0001-8983-3049

Universidade Anhembi Morumbi, Brasil

E-mail: luiza.lunardi@gmail.com

Camila Robbi Semeghin

ORCID: https://orcid.org/0000-0002-2553-1102 Universidade Anhembi Morumbi, Brasil

E-mail: camilarobbi@hotmail.com

Bruna Kelly Sousa Hirata

ORCID: https://orcid.org/0000-0002-5774-8955 Universidade Anhembi Morumbi, Brasil

E-mail: bruna.hirata@anhembi.br

\begin{abstract}
Resumo
O ano de 2021 foi marcante para os pacientes diagnosticados com diabetes e para a medicina, pois completou-se os 100 anos de descoberta da insulina exógena. Este medicamento revolucionou a qualidade de vida de milhares de pessoas, além de proporcionar para a medicina e indústria farmacêutica, uma nova perspectiva. Nesse contexto, é importante evidenciar que o diabetes mellitus tipo 1 é uma doença autoimune, onde o próprio organismo ataca as células produtoras de insulina, as células beta pancreáticas, sendo assim, é essencial para esses pacientes a utilização desta substância de forma exógena durante toda a vida. Atualmente, existem diversos tipos de insulinas e tecnologias em constante evolução que visam facilitar a adesão e promover maior bem-estar a essas pessoas. Tendo isso em vista, esta revisão literária tem como objetivo mostrar mais sobre os tipos de diabetes que existem, as possíveis causas, os tratamentos existentes, as consequências da falta de controle da glicose, a descoberta da insulina, as tecnologias e uma breve comparação entre o sistema de saúde brasileiro ao sistema de saúde estadunidense. Utilizando materiais bibliográficos como fonte de pesquisa, esse artigo trata-se de um artigo descritivo sobre o diabetes tipo 1, que visto as estimativas e atuais prevalências, torna-se indispensável a discussão sobre essa doença.
\end{abstract}

Palavras-chave: Diabetes tipo 1; Insulinas; Tratamento do diabetes tipo 1.

\begin{abstract}
The year of 2021 was a milestone for medicine and especially for patients diagnosed with diabetes, as it completed 100 years of the discovery of exogenous insulin. This medicine has completely revolutionized thousands of people's quality of life, as well as providing healthcare and the pharmaceutical industry a new perspective. In this context, it is important to show that type 1 diabetes mellitus is an autoimmune disease, meaning that the body itself attacks insulin-producing cells, called beta cells, therefore, it is essential that these patients use this hormone exogenously throughout their entire life's period. Currently, there are several types of insulin and technologies in constant development that aim to facilitate adherence and promote greater well-being for these people. Considering that the present literary review aims to describe the main types of diabetes, possible causes, existing treatments, consequences of lack of glucose control, the discovery of insulin, technologies and a brief comparison between the United States' and the Brazilian healthcare system. Using bibliographic materials as a research source, this article is a descriptive article about type 1 diabetes, which, considering the estimates and current prevalence, makes the discussion about this disease indispensable.
\end{abstract}

Keywords: Type 1 diabetes; Insulin; Therapy of type 1 diabetes.

\section{Resumen}

El año 2021 fue un hito para los pacientes diagnosticados de diabetes y para la medicina, ya que se cumplió el centenario del descubrimiento de la insulina exógena. Este medicamento revolucionó la calidad de vida de miles de personas, además de aportar una nueva perspectiva a la medicina y a la industria farmacéutica. En este contexto, es importante destacar que la diabetes mellitus tipo 1 es una enfermedad autoinmune, en la que el propio organismo ataca a las células 
productoras de insulina, las células beta del páncreas, por lo que es imprescindible que estos pacientes utilicen esta sustancia de forma exógena durante toda su vida. Actualmente, existen varios tipos de insulinas y tecnologías en constante evolución que pretenden facilitar la adherencia y promover un mayor bienestar a estas personas. Con esto en mente, esta revisión bibliográfica pretende mostrar más sobre los tipos de diabetes que existen, las posibles causas, los tratamientos existentes, las consecuencias de la falta de control de la glucosa, el descubrimiento de la insulina, las tecnologías y una breve comparación entre el sistema sanitario brasileño y el estadounidense. Utilizando material bibliográfico como fuente de investigación, este artículo es un artículo descriptivo sobre la diabetes tipo 1, que, dadas las estimaciones y la prevalencia actual, se hace imprescindible para hablar de esta enfermedad.

Palabras clave: Diabetes tipo 1; Insulinas; Tratamiento de la diabetes tipo 1.

\section{Introdução}

O Diabetes Mellitus (DM) é uma doença metabólica crônica caracterizada por hiperglicemia persistente, por decorrência da ausência ou deficiência da produção/secreção da insulina, a qual exerce um papel central na regularização da homeostase da glicose no sangue, também podendo se manifestar por meio da resistência à ação da insulina (Van Belle; Coppieters; Von Herrath, 2011). Segundo os dados da 9a edição do Atlas de Diabetes, atualmente cerca de 463 milhões de adultos possuem diabetes ao redor do mundo (IDF, 2021), enquanto no Brasil, a estimativa dos dados da Sociedade Brasileira de Diabetes é de que mais de 12 milhões de brasileiros vivem com a doença (Damasceno, 2021). Estima-se que em 2040 mais de 642 milhões de pessoas terão diabetes (International Diabetes Federation (IDF). Diabetes Atlas $7^{a}$ ed. Brussel: IDF; 2015). Esse aumento significativo na sua prevalência está relacionado com as alterações alimentares, sedentarismo, aumento da expectativa de vida e maior sobrevida daqueles com a doença (SBD, 2019).

Popularmente, o DM é conhecido por ter dois tipos, porém, de acordo com as Diretrizes da Sociedade Brasileira de Diabetes, existem outros tipos:

- DM Tipo 1 (DM1): decorrente de destruição autoimune das células beta pancreáticas, poligênica, hereditário que acomete de 5 a $10 \%$ dos brasileiros com diabetes, no qual pouca ou nenhuma insulina é liberada no corpo. Os portadores da doença são insulino - dependentes e é diagnosticada geralmente na infância ou adolescência SBD, 2019).

- DM Tipo 2: estado no qual o corpo não aproveita de forma correta a insulina que foi produzida, caracterizada pelo estado de resistência à insulina. Em geral, está ligado ao excesso de peso, hábitos alimentares e sedentarismo, representando cerca de 90\% dos casos de DM no Brasil, tendo sua maior incidência em adultos (WU, DING, TANAKA, \& ZHANG, 2014).

- DM Gestacional: ocorre durante a gravidez e de forma temporária. As taxas glicêmicas ficam acima do normal durante a gestação, com ausência de DM prévia e é explicada pelo aumento de hormônios contrarreguladores da insulina dado pelo estresse fisiológico que uma gravidez causa, além de fatores genéticos ou ambientais. O principal hormônio relacionado é o Hormônio lactogênico placentário (HLP) que estimula o desenvolvimento da mama e da lactação, porém, também causa resistência à ação da insulina, resultando na redução da captação de glicose nos tecidos maternos para que seja disponibilizado mais energia para o feto (Blotta, 2018).

- DM Neonatal: é definido como a presença de hiperglicemia nos primeiros três meses de vida (SBD, 2019) podendo ser transitório (DNT) ou permanente (DNP), sendo a segunda mais frequente.

- MODY (maturity-onset diabetes of the Young): é definido como um tipo de diabetes familiar de transmissão autossômica-dominante. O diagnóstico é feito durante a infância, adolescência ou em adultos jovens, mas o que é levado em conta para que o indivíduo seja diagnosticado com esse tipo de DM, é o histórico familiar. A presença de três gerações da mesma linhagem afetadas pela doença é o que a diferencia da DM1.

- DM tipo LADA (Latente Autoimune do Adulto): representa aproximadamente 2\% da população e expressa o agravamento do tipo 1 em adultos. É acarretada por um processo autoimune do organismo que ataca as células do pâncreas (SBD, 2019).

Apesar de mais de $90 \%$ dos casos de diabetes ser do tipo 2 (Wu, Ding, Tanaka, \& Zhang, 2014), o DM1 também possui 
uma parcela significante. Ao contrário do diabetes tipo 2, que por algumas vezes pode ser evitado por meio dos hábitos de vida, o DM1 possui uma grande carga genética. Esse artigo trará mais informações sobre o DM1 e, por isso, daremos uma revisada em alguns conceitos sobre ele.

\section{Diabetes mellitus tipo 1}

Ocorre pela falta de produção da insulina em decorrência da destruição autoimune das células betas das ilhotas pancreáticas. Essa destruição ainda não é totalmente compreendida, mas sabe-se que ocorre por interações entre genes de suscetibilidade, autoantígenos e fatores ambientais.

Os genes de suscetibilidade são aqueles que pertencem ao complexo principal de histocompatibilidade (MHC), sendo identificados em mais de 90\% dos pacientes com DM1, como HLA-DR3, DQB1*0201 e HLA-DR4, DQB1*0302 (Brutsaert, 2020).

Os autoantígenos ainda não possuem mecanismos de ação claros, mas acredita-se que são provenientes de lesões das células beta pancreáticas, que são reconhecidas pelo sistema imune e induz o processo de autoimunidade órgão-específico (Almeida de Sousa, Caetano Albernaz, \& da Rocha Sobrinho, 2016).

Os fatores ambientais incluem infecções por vírus, como coxsackie, rubéola, citomegalovírus, Epstein-Barr e retrovírus, também foram ligados ao desenvolvimento do DM1, uma vez que eles podem infectar e destruir diretamente ou indiretamente as células-beta. Além disso, a dieta também pode contribuir para o desenvolvimento da doença, como o consumo de derivados lácteos das lactantes, água muito rica em nitratos, baixo consumo de vitamina D e exposição ao glúten e a cereais no primeiro ano de vida (Brutsaert, 2020).

\section{Diagnóstico}

O diagnóstico do DM1 deve ser realizado utilizando critérios como glicemia em jejum, teste oral de tolerância à glicose (TOTG) e hemoglobina glicada (HbA1c). A glicemia de jejum é realizada por meio de coleta de sangue após um jejum de no mínimo 8 horas, enquanto o TOTG é feito a partir de uma coleta inicial de sangue em jejum, seguida de uma ingestão de $75 \mathrm{~g}$ de glicose e novas coletas durante o intervalo de 2 horas. O exame da hemoglobina glicada (HbA1c) mostra os níveis de glicação da hemoglobina causados pela hiperglicemia persistente, e desta forma, permite avaliar alterações dos níveis glicêmicos dos últimos 3 a 4 meses. O exame apresenta vantagem por sofrer menor variabilidade no ao longo do dia e independer do jejum (MENDES e DIEHL, 2019).

Para que o diagnóstico seja confirmado, os exames precisam ser refeitos e permaneceram alterados, seguindo os valores representados na tabela 1 (Liga Interdisciplinar de Diabetes, 2017).

Tabela 1 - Critérios laboratoriais para diagnóstico da DM (SBD, 2019-2020).

\begin{tabular}{|c|c|c|c|c|}
\hline & $\begin{array}{c}\text { Glicose em jejum } \\
(\mathbf{m g} / \mathbf{d L})\end{array}$ & TOTG (mg/dL) & $\begin{array}{c}\text { Glicose ao acaso } \\
(\mathbf{m g} / \mathbf{d L})\end{array}$ & HbA1C (\%) \\
\hline Normoglicemia & $<100^{* *}$ & $<140$ & - & $<5,7$ \\
\hline $\begin{array}{c}\text { Pré-diabetes ou } \\
\text { risco aumentado } \\
\text { para DM }\end{array}$ & $\geq 100 \mathrm{e}<126$ & $\geq 140 \mathrm{e}<200$ & - & $\geq 5,7 \mathrm{e}<6,5$ \\
\hline $\begin{array}{c}\text { Diabetes } \\
\text { estabelecido }\end{array}$ & $\geq 126$ & $\geq 200$ & $\begin{array}{c}\geq 200 \mathrm{com} \\
\text { sintomas de } \\
\text { hiperglicemia }\end{array}$ & $\geq 6,5$ \\
\hline
\end{tabular}

*Critérios laboratoriais adotados pela SBD. **A OMS considera normalidade na glicose em jejum até 120mg/dL Fonte: Autores. 


\section{Manifestações clínicas do DM1}

Devido ao estado catabólico promovido pela ausência de insulina, as principais manifestações do DM1 são a descompensação metabólica com poliúria, polidipsia, emagrecimento e cetoacidose.

Polidipsia (sede excessiva) e poliúria (excesso de urina) são os dois sintomas mais conhecidos e são consequências do estado de hiperglicemia, uma vez que os rins não conseguem reabsorver toda a glicose presente, a qual acaba sendo eliminada pela urina, junto de uma grande quantidade de água, explicando também a desidratação e causando sede excessiva (Barbosa Murro, Tambascia, \& de Carvalho Ramos, 2011)

O acentuado emagrecimento se dá por conta da falta de energia causada pela ausência de insulina, levando as células insulino-dependentes a utilizarem as reservas lipídicas presentes no tecido adiposo. Este quadro também favorece o desenvolvimento de cetoacidose. Quando a única fonte de energia passa a ser ácidos graxos, inicia-se no tecido adiposo a produção de compostos chamados corpos cetônicos, os quais suprem a falta de energia no coração, músculos esqueléticos, rins e cérebro. Entretanto, há alteração no equilíbrio iônico do sangue, reduzindo o pH sanguíneo. A cetoacidose, quando não tratada, é considerada uma emergência, podendo levar o paciente ao coma e até a morte (Brutsaert, 2020). Pode ser necessária uma internação e administração de grandes quantidades de líquido, juntamente com eletrólitos como sódio, potássio, cloro e fosfato, para repor tudo que foi perdido na micção excessiva.

\section{Possíveis Complicações Associadas}

Dentre as possíveis complicações que o paciente portador de DM1 não controlada pode ter, existem dois grandes grupos: as microvasculares e as macrovasculares.

No grupo das complicações microvasculares, encontramos a nefropatia diabética, que é responsável por pelo menos metade dos casos de insuficiência renal e é marcada pela presença de albumina na urina que, dependendo da quantidade, é denominado microalbuminúria ou macroalbuminúria; a retinopatia diabética que é uma alteração nos vasos sanguíneos que nutrem a retina que causam o fechamento progressivo de suas paredes, causando microaneurismas que podem ocasionar em hemorragias, levando a cegueira; e por último, a neuropatia diabética, que é a presença de sintomas e/ou sinais de disfunção dos nervos periféricos, podendo se manifestar de várias formas, desde síndromes dolorosas, agudas, graves e secundárias a formas assintomáticas (Brutsaert, 2020).

Já no grupo das macrovasculares, temos as doenças cardiovasculares que podem atingir o coração, cérebro e membros inferiores. A hiperglicemia é apenas um dos mecanismos que aumentam o risco da doença macroyascular no diabético, a hipertensão, dislipidemia e fumo também estão relacionados. O pé diabético é uma consequência das alterações vasculares nos membros inferiores., O Grupo Internacional sobre Pé Diabético (INGDF) relata que de todas as amputações não traumáticas de membros inferiores, $40 \%$ a $60 \%$ são realizadas em pacientes com diabetes (Brutsaert, 2020).

\section{Metodologia}

A revisão qualitativa da literatura teve como base artigos científicos publicados a partir do ano 2010, com uma exceção, na qual o artigo foi publicado em 1922. Foram utilizadas bases de dados PubMed, mantida pela Biblioteca National de Medicina (EUA), Scientific Eletronic Library Online (SciELO) e Google Scholar com o uso dos descritores: "type 1 diabetes”, "Multiple Daily Injections", “continuous glucose monitoring”, “insulin pump”, “t1d” “100 years insulin”. "Inhaled insulin”, "Frederick Banting”, "NPH insulin", “diabetes technology", “insulin discovery”, “diabetic neuropathies”, "complications in diabetic patients", "diabetic foot" e "tld diagnosis".

Foram usados como critérios de inclusão: artigos disponíveis na íntegra nas bases de dados selecionadas, artigos disponíveis nos idiomas inglês e português, livros, sites de associações de diabetes, banco de imagens da UNESCO. 
Os critérios de exclusão foram: os artigos em duplicidade, estudos que incluíam medicações orais, dietas e indivíduos com outro tipo de diabetes foram excluídos.

Toda revisão literária precisa de estudos bibliográficos, visando mapear os trabalhos que abordam uma determinada temática de interesse dos autores (Gil, 2002). Por meio deste mapeamento, foi possível fazer um levantamento dos anos em que os artigos utilizados foram publicados, conforme a Tabela 2.

Tabela 2 - Relação do ano de publicação dos artigos utilizados na revisão literária.

\begin{tabular}{|c|c|}
\hline Ano de publicação & $\begin{array}{c}\text { Artigos utilizados } \\
\text { (quantidade) }\end{array}$ \\
\hline 2021 & 7 \\
\hline 2020 & 6 \\
\hline 2019 & 7 \\
\hline 2018 & 1 \\
\hline 2017 & 1 \\
\hline 2016 & 2 \\
\hline 2015 & 1 \\
\hline 2014 & 7 \\
\hline 2013 & 1 \\
\hline 2012 & 2 \\
\hline 2011 & 3 \\
\hline 2010 & 1 \\
\hline Anteriores à 2010 & 7 \\
\hline Sem data & 4 \\
\hline
\end{tabular}

Fonte: Autores.

\section{Resultados e Discussão}

\section{Insulina e sua descoberta}

A insulina é um hormônio produzido pelo pâncreas que tem como função metabolizar a glicose, atuando como uma espécie de "chave" que auxilia a entrada da glicose nas células para a produção de energia (Petersen \& Shulman, 2018). No DM1 há disfunção em sua produção, fazendo-se necessário a utilização de aplicações diárias.

A descoberta da insulina foi um grande marco na história da medicina. Antes de sua descoberta, o diagnóstico de diabetes era considerado uma sentença de morte. As dietas restritivas eram a luz no fim do túnel, mesmo sendo radicais e ineficazes. Os pacientes chegavam a ingerir menos de $1000 \mathrm{kcal}$ ao dia, e mesmo assim continuavam tendo os níveis de glicose sanguíneos acima do esperado (Ross \& Neville, 2019). Todavia, sua descoberta trouxe uma nova chance de vida a todos os pacientes diagnosticados. 
Figura 1: Dr. Banting e um dos cães que foram usados durante os testes.

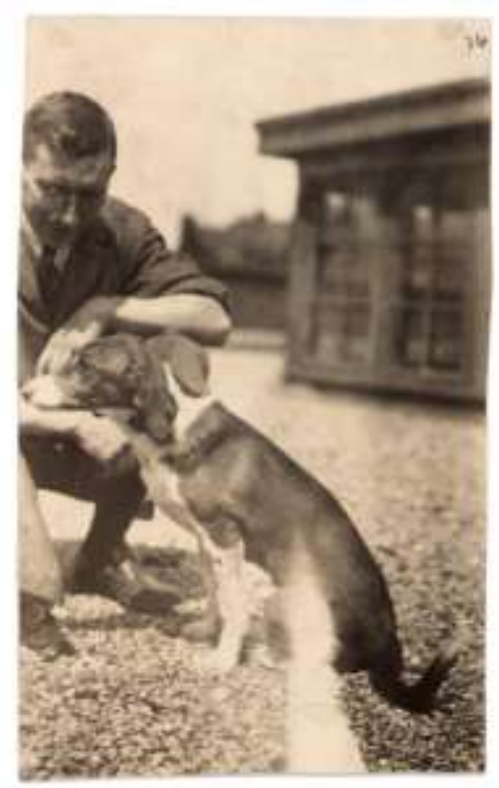

Fonte: University of Toronto Library (2021).

Em meados de 1920, com base em um artigo publicado por Moses Barron, no qual deduzia que as ilhotas pancreáticas secretariam uma substância na corrente sanguínea que controlaria os níveis glicêmicos. Frederick Banting, retratado na figura 1, após ler este estudo, concluiu que se conseguisse isolar este hormônio liberado pelas ilhotas, poderia salvar os pacientes com diabetes tipo 1 (Hegele, 2020). Banting concluiu que ao ligar o ducto principal do pâncreas, poderia aguardar as células acinares serem destruídas e conseguiria interromper a produção das enzimas digestivas e preservar as ilhotas, podendo extrair a molécula que controlava a glicemia (Hegele, 2020).

Em 1921, Dr. Banting, junto de seu estudante Charles Best, iniciaram os estudos no Departamento de Fisiologia da Universidade de Toronto. Utilizando cães para testar o hormônio isolado, os pesquisadores o administraram 1x por dia durante 4 dias e observaram que a glicemia estava diminuindo e as condições dos animais estavam melhorando. Essa preparação foi nomeada "Isletin", mais tarde chamada de "insulina" (Hegele, 2020).

O primeiro paciente humano a injetar o extrato purificado de insulina foi o Leonard Thompson, um menino de 14 anos de idade, retratado na figura 2. Leonard sobrevivia com diabetes há 2 anos e meio quando foi internado no hospital geral de Toronto, pesando apenas 29,48 kg com sintomas de cetoacidose diabética (Ross e Neville, 2019). Apesar da dieta de apenas 450 kcal/dia seus sintomas só pioravam. Levando em consideração o quadro, seu pai concordou com a participação em um estudo clínico em que seria administrado um extrato de insulina bovina de forma intravenosa. Leonard recebeu a primeira dose de insulina no dia 11 de janeiro de 1922. (Banting et al., 1922) Sua glicemia teve redução de 25\% em 24h, entretanto, devido a um abcesso por reações alérgicas, o tratamento foi descontinuado. Doze dias depois, Leonard recebeu um novo extrato purificado (Hegele, 2020). Em 24h, sua glicemia teve uma redução de 77\%. Leonard Thompson teve alta do hospital em que estava no dia 15 de maio de 1922 sem o uso de insulina, voltando à dieta restritiva, o que resultou em retorno dos sintomas iniciais. Os médicos então concluíram que o uso da insulina deveria ser diário e por toda a vida (Ross e Neville, 2019). 
Figura 2: Leonard Thompson, primeiro paciente a receber insulina.

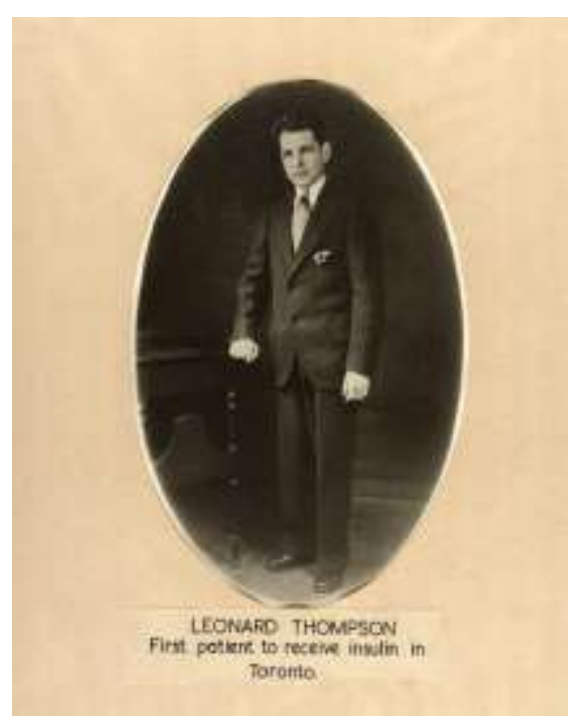

Fonte: University of Toronto Library (2021).

Após a descoberta revolucionária feita por Banting, a insulina precisou ser purificada para que houvesse menores reações de pele. John James Richard Macleod e James Bertram Collip ajudaram neste processo. Juntos, eles ganharam o Nobel de Fisiologia e Medicina em 1923 (Hegele, 2020).

\section{Insulina e os dias de hoje}

Após 100 anos de sua revolucionária descoberta, a insulina passou por muitas revoluções tecnológicas. Ao final de 1923, empresas farmacêuticas Eli Lilly and Company e Nordisk Laboratory começaram sua produção utilizando pâncreas suínos. Eram necessários cerca de $80 \mathrm{~kg}$ de pâncreas para a produção de $1 \mathrm{mg}$ de insulina que necessitava ser purificada. Em meados de 1980, com o advento da biologia molecular, houve o desenvolvimento da insulina humana, a qual utilizava a tecnologia de DNA recombinante. Tal insulina apresenta absorção mais rápida e período de ação mais curto. Em 2000, os análogos de insulina humana foram aprovados pela Food and Drugs Administration (FDA) e European Medicines Evaluation Agency (EMEA). Os análogos apresentam alterações na sua cadeia de aminoácidos, trazendo melhorias no seu tempo de ação. (Retnakaran et al, 2021).

As alterações na cadeia da insulina consistem em substituição ou remoção de resíduos ácidos nas cadeias A ou B e, em alguns casos, existe a possibilidade de inserir cadeias. A insulina detemir, por exemplo, houve a remoção do resíduo de treonina na cadeia B30 e a adição do ácido tetradecanoico no resíduo da lisina não cadeia B29. Está mínima alteração tornou a absorção mais longa (Tibaldi, 2014).

Em 1936, a primeira insulina de ação intermediária foi produzida por Hans Christian Hagedorn e seus colegas. Após 10 anos e algumas melhorias, introduziram a insulina Neutral protamine Hagedorn (NPH) (Owens, 2011), a qual era obtida por meio da precipitação da insulina humana recombinante sintetizada com zinco, na presença de protamina (Saleem \& Sharma, 2021).

\section{Tipos de insulina disponíveis}

Os seres humanos com função pancreática normal produzem insulina de forma a suprir as necessidades fisiológicas, tendo o tempo de ação inicial, pico e tempo de efeito especificamente exatos. Quando a insulina é injetada no corpo, as moléculas formam hexâmeros que se difundem pelo fluido intersticial e penetram nas paredes capilares, entrando então na corrente 
sanguínea. Tendo em vista o tempo que esse processo pode levar, foram criadas diferentes formulações com tempo de início de ação, pico e duração de efeitos diferentes, tendo como objetivo atender as necessidades específicas de cada paciente (Ahmad, 2014).

\section{Insulina basal}

O fígado, além de suas outras muitas funções, armazena glicose de uma forma compacta, conhecida como glicogênio e secretada na corrente sanguínea de forma que esteja provendo uma constante fonte de energia para os órgãos vitais e os tecidos. (Scheiner, 2020) Pensando em um indivíduo que não tem diabetes, temos o equilíbrio das funções pancreáticas, em especial a produção de insulina, em conjunto com a produção e armazenamento hepático de glicogênio. A homeostase glicêmica é mantida pelo fígado, que deixa o glicogênio em equilíbrio com as quantidades de insulina liberadas pelo pâncreas. Um indivíduo portador de DM1 precisa suprir essa quantidade de insulina que é secretada ao longo do dia, também conhecida como insulina basal.

A insulina basal pode ser apresentada de diversas formas. A insulina de ação intermediária (NPH) chega à corrente sanguínea após 2-4h e tem pico de ação entre 4-12h depois, sendo efetiva por até 12-18h. Este tipo de insulina basal não se enquadra às necessidades do corpo, raramente mantém níveis de glicose estáveis, principalmente durante o dia (Scheiner, 2020). As insulinas basais de longa duração (Glargina, Degludeca e Detemir) podem ser administradas uma ou duas vezes ao dia, chegam à corrente sanguínea algumas horas após a injeção e tendem a baixar os níveis de glicose sanguínea por até 24h. As insulinas basais de duração ultralonga (Toujeo) chegam à corrente sanguínea após 6h, não tem pico e podem durar mais de 36h (ADA).

\section{Insulina Bolus}

Após a ingestão de carboidratos, a glicemia tende a se elevar de forma rápida, tendo início após 15 minutos e um pico entre 30-90 minutos. Portanto, para manter a glicose sanguínea dentro do alvo, é necessário que haja picos de insulina para estabilizá-la. Esses picos de insulina são conhecidos como insulina bolus (Malik et al., 2014). A insulina bolus é dividida em 2 grupos: rápida e regular. A insulina de ação rápida (Asparte, Lispro e Glulisina) tem o início de sua ação 15 minutos após injetada, apresentando pico de ação após 1-2h e tempo de duração de 2-4h. A insulina regular ou de curta duração (Humulin $\mathrm{R}$ ®, Novolin R ${ }^{\circledR}$ e Velosulin R ${ }^{\circledR}$ ) chega na corrente sanguínea 30 minutos após injetada, tendo pico após 2-3h e permanece no corpo até $6 \mathrm{~h}(\mathrm{ADA})$.

Neste grupo, ainda pode ser citada a insulina inalável, que é uma alternativa relativamente nova no mercado e demonstrou ser segura e de fácil administração. É comercializada em blisters de 1 e $3 \mathrm{mg}$ (e cada blister de $1 \mathrm{mg}$ equivale a 3 UI de insulina regular). A insulina inalável tem absorção e pico de ação mais rápidos do que os análogos de ação rápida, tendo em vista que o pulmão é extremamente vascularizado (Chan J et al., 2017)

Os grupos citados apresentam uma média de início de ação, pico e duração, entretanto, os valores variam entre cada tipo de insulina. Sendo assim, um paciente portador de diabetes tipo 1 tem a necessidade de administrar dois tipos de insulina, uma para manter a quantidade de insulina basal que o corpo deveria secretar e outra para corrigir as refeições realizadas ao longo do dia.

\section{Insulinoterapia}

O tratamento do DM1 consiste na administração de insulina exógena durante toda a vida, juntamente com a monitorização da glicemia (Malik et al., 2014). A administração da insulina pode ser feita de duas formas, sendo pela Bomba de Infusão de Insulina ou pelas Múltiplas Injeções Diárias, também conhecida como MDI (Multiple Daily Injections) (Ross e Neville, 2019). MDI, como o próprio nome diz, consistem em injeções manuais periódicas a fim de suprir as necessidades do 
organismo simulando a atividade que o pâncreas faria em condições normais. A insulina inalável pode ser inserida neste cenário, substituindo apenas as insulinas bolus, tendo a necessidade de manter os níveis basais em injeções (Kim e Plosker, 2015).

As bombas de insulina liberam pequenas quantidades de insulina de rápida absorção durante o dia e a noite, como uma tentativa de imitar a secreção fisiológica (Nimri et al., 2020). Com ela, os níveis de insulina basal podem ser ajustados de acordo com as necessidades específicas do corpo. A terapia com bomba de insulina pode proporcionar uma melhor qualidade de vida ao paciente e à sua família, sendo muito mais flexível e tendo melhora significativa em quadros de depressão, ansiedade, aumento de autoestima e de responsabilidade com o tratamento. Atualmente, existem diversas bombas de insulina no mercado. Algumas, são do modo convencional em que há um tubo que liga a bomba à uma cânula no corpo. Mas também existem as chamadas "Patch", na qual a bomba fica grudada à pele. Algumas bombas têm integração com sensores de monitoramento contínuo da glicose (CGM), outras têm a necessidade de incluir manualmente o valor da glicemia e a quantidade a ser tomada de insulina. É um processo constante de evolução, tendo grandes atualizações nos últimos anos.

Para indivíduos com DM (de qualquer tipo), é de extrema importância ter controle dos valores glicêmicos para conseguir ajustar a quantidade de insulina a ser tomada, tanto na forma basal quanto a bolus. Nos últimos anos, os glicosímetros têm tido grande evolução, em conjunto com as bombas de insulina. Fomos de tiras de urina para sensores que medem a glicemia a cada 5 minutos (Cappon et al., 2019). Em 1965, foi criado o primeiro glicosímetro, chamado de Dextrostix (Umpierrez, 2018). Consistia em uma fita que, por meio de glicose oxidase, mudava a cor e era comparada a uma escala pré-estabelecida. Apenas médicos usavam. Hoje em dia, é essencial que todos os diabéticos tenham um glicosímetro em casa (Cappon et al., 2019). O automonitoramento da glicemia se tornou uma forma de cuidado pessoal para os pacientes. Este avanço, em conjunto com o exame laboratorial de HbA1c e o uso de insulinoterapia, possibilitou o controle da doença e diminuição (ou controle) das complicações (Advani, 2020).

As bombas de insulina tiveram uma evolução rápida. Em 40 anos, ficaram menores, mais precisas e mais confiáveis (Kravarusic et al., 2020). A primeira bomba de insulina foi criada em 1963 por Arnold Kardish, era usada apenas para estudos clínicos e liberava tanto insulina quanto glucagon. Foi o primeiro sistema de pâncreas artificial criado, o qual continha uma grande bomba de insulina com um analisador, que media a quantidade de açúcar no sangue com um mecanismo que controla as funções da bomba quando a glicose estava alterada (ALSALEH et al., 2010). Era tão grande que parecia uma mochila escolar. Aprovada em 2012 (FDA) e evoluindo desde então, a bomba de infusão de insulina OmniPod é a forma mais atual de "Patch". Da empresa Insulet, esta bomba não apresenta nenhum tubo, funciona por wireless e o "Pod" dura até 3 dias grudado ao corpo, injetando microdoses de insulina durante este tempo, como podemos ver na Figura 3.

Figura 3: Bomba de insulina Omni Pod.

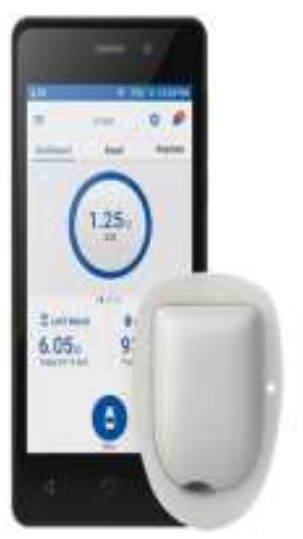

Fonte: https://www.omnipod.com/en-ca. 
Comercializada desde 2010 no Brasil, a AccuChek Spirit Combo da Roche é um sistema de infusão contínua de insulina que se assemelha ao funcionamento de um pâncreas, tendo a necessidade de medir a glicemia de forma capilar e cadastrar manualmente a quantidade de carboidratos ingeridos para liberação das doses de insulina. Não tem correlação com sensores de glicose (https://www.accu-chek.com.br/br/ids/index.html). Aprovada em 2016 (FDA), a MiniMed 670G da Medtronic é a bomba de insulina mais recente disponível no Brasil, sendo considerado um sistema de pâncreas artificial. Em conjunto com a tecnologia SmartGuard, pode ser programada a liberação de insulina basal tendo como base os valores do sensor de monitoramento de glicose contínua, e pode ser suspendida quando a glicemia baixar. Aprovada recentemente (FDA), T:slim X2 da Tandem Diabetes Care contém uma tecnologia Basal-IQ que pode suspender a liberação de insulina de acordo com as leituras de glicemia do sensor de glicose continua (normalmente o Dexcom 5). Esta, em especial, não está disponível no Brasil até o momento. As figuras abaixo correspondem às bombas de insulina citadas, respectivamente.

Figura 4: Bomba de insulina accu-check spirit combo.

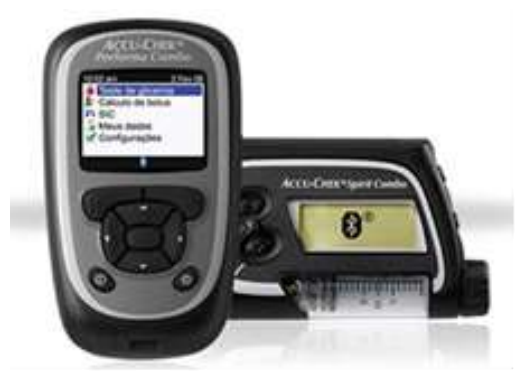

Fonte: https://www.accu-chek.com.br/br/ids/combo/

Bomba de insulina minimed $670 \mathrm{~g}$

Figura 5: Bomba de insulina minimed 670g.

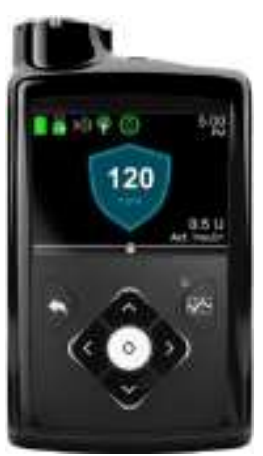

Fonte: https://www.medtronicdiabetes.com/customer-support/minimed-670g-system-support. 
Figura 6: Bomba de insulina t:slim x2.

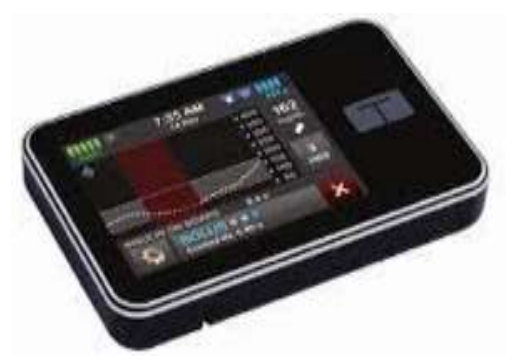

Fonte: https://www.tandemdiabetes.com/products/t-slim-x2-insulin-pump.

\section{A insulinoterapia do DM1 no Brasil}

No Brasil é possível adquirir bomba de insulina por meio do Sistema Único de Saúde (SUS), levando em consideração o artigo $5^{\circ}$ e 196 da Constituição Federal, o qual assegura que a saúde é um direito de todos e o Estado tem como obrigação promover os meios para que isso aconteça, garantindo acesso às ações e serviços para que isso ocorra, dando assistência terapêutica integral, inclusive a farmacêutica (Lei $\mathrm{n}^{\circ}$ 8.080, art 6 $6^{\circ}$, , “d”). Neste cenário, os pacientes com diabetes conseguem pelo SUS não apenas a bomba de insulina (sendo neste caso via ordem judicial), mas todos os insumos do tratamento. Ou seja, é possível conseguir as insulinas, fitas, glicosímetro e agulhas. (https://www.gov.br/saude/pt-br)

O SUS é um grande diferencial no sistema de saúde brasileiro. Nos Estados Unidos, é comum ver pacientes com diabetes fracionando as doses de insulina para que a ampola dure alguns dias a mais, pois além de muito cara, nem todos possuem plano de saúde para ter descontos ao comprar as medicações que precisam para viver (Pronin, 2019). Apesar do país possuir inflação baixa, os valores ficaram $300 \%$ mais caros nos últimos dez anos. Segundo uma pesquisa recente da GoodRx, empresa que rastreia preços de farmácias, (AFP, 2020) (aproximadamente R \$1.700), enquanto o relatório da T1International mostra que esse mesmo frasco custava US\$130 em 2012 (ou R \$700). No Brasil, esse mesmo produto pode ser comprado pelo preço máximo estipulado pelo governo de $\mathrm{R} \$ 125,30$, de acordo com a Eli Lilly ou, ainda, encontrado no SUS, onde é de graça (Pronin, 2019).

\section{Conclusão}

O diabetes, sendo uma doença autoimune, pode acometer qualquer pessoa de qualquer faixa etária, portanto, é de extrema importância que os tratamentos continuem evoluindo. Dentre os tratamentos existentes, vale ressaltar a insulina, um hormônio de uso indispensável para manter a qualidade de vida dos pacientes, além de evitar possíveis complicações da doença a longo prazo, como neuropatias.

Atualmente essas substâncias estão passando por constantes inovações e, hoje em dia, existe uma grande variedade de opções, tudo isso graças a sua descoberta há 100 anos. As novas tecnologias visam facilitar a adesão ao tratamento para estabelecer maior bem-estar na vida dos pacientes. Além disso, o controle glicêmico é de extrema importância, visto que tem grande influência na homeostase de diversos sistemas corporais.

Levando em consideração o crescimento da quantidade de pacientes com DM, o aprimoramento das tecnologias no tratamento é uma realidade não tão distante. Entretanto, é de extrema importância que os médicos e cientistas continuem realizando pesquisas a fim de expandir os conhecimentos em relação à patologia, visando melhorar a qualidade de vida dos pacientes. 


\section{Referências}

A. F. P. (2020). Insulina, vital para diabéticos, tem mercado ilegal e gratuito nos EUA. Exame. https://exame.com/ciencia/insulina-vital-para-diabeticos-temmercado-negro-e-gratuito-nos-eua

Advani A. (2020). Positioning time in range in diabetes management. Diabetologia, 63(2), 242-252. https://doi.org/10.1007/s00125-019-05027-0

Ahmad K. (2014). Insulin sources and types: a review of insulin in terms of its mode on diabetes mellitus. Journal of traditional Chinese medicine $=$ Chung $i$ tsa chih ying wen pan, 34(2), 234-237. https://doi.org/10.1016/s0254-6272(14)60084-4

Almeida de Sousa, A., Caetano Albernaz, A., \& da Rocha Sobrinho, H. M. (2016). Diabetes Melito tipo 1 autoimune: aspectos imunológicos.

Alsaleh, F. M., Smith, F. J., Keady, S., \& Taylor, K. M. (2010). Insulin pumps: from inception to the present and toward the future. Journal of clinical pharmacy and therapeutics, 35(2), 127-138. https://doi.org/10.1111/j.1365-2710.2009.01048.x

American Diabetes Association (2012). Diagnosis and classification of diabetes mellitus. Diabetes care, 35 Suppl 1(Suppl 1), S64-S71. https://doi.org/10.2337/dc12-s064

Andersen, A. R., Christiansen, J. S., Andersen, J. K., Kreiner, S., \& Deckert, T. (1983). Diabetic nephropathy in Type 1 (insulin-dependent) diabetes: an epidemiological study. Diabetologia, 25(6), 496-501. https://doi.org/10.1007/BF00284458

Atkinson, M. A., Eisenbarth, G. S., \& Michels, A. W. (2014). Type 1 diabetes. Lancet (London, England), 383(9911), 69-82. https://doi.org/10.1016/S01406736(13)60591-7

Banting, F. G., Best, C. H., Collip, J. B., Campbell, W. R., \& Fletcher, A. A. (1922). Pancreatic Extracts in the Treatment of Diabetes Mellitus. Canadian Medical Association journal, 12(3), 141-146.

Banting, F. G., Sir, 1891-1941. (1920). Note dated Oct 31/20 from loose leaf notebook 1920/21. insulin: N10002. https://insulin.library.utoronto.ca/islandora/object/insulin\%3AN10002

Banting, F. G., Sir, 1891-1941. (1922). Photograph of Banting with dog ca. https://insulin.library.utoronto.ca/islandora/object/insulin:S10001

Blair, J. C., McKay, A., Ridyard, C., Thornborough, K., Bedson, E., Peak, M., Didi, M., Annan, F., Gregory, J. W., Hughes, D. A., Gamble, C., \& SCIPI investigators (2019). Continuous subcutaneous insulin infusion versus multiple daily injection regimens in children and young people at diagnosis of type 1 diabetes: pragmatic randomised controlled trial and economic evaluation. BMJ (Clinical research ed.), 365, 11226. https://doi.org/10.1136/bmj.11226

Boulton, A. J., Gries, F. A., \& Jervell, J. A. (1998). Guidelines for the diagnosis and outpatient management of diabetic peripheral neuropathy. Diabetic medicine: a journal of the British Diabetic Association, 15(6), 508-514. https://doi.org/10.1002/(SICI)1096-9136(199806)15:6<508::AID-DIA613>3.0.CO;2-L

Brasil. Lei $N^{o}$ 8.080, De 19 De Setembro De 1990. http://www.planalto.gov.br/ccivil_03/leis/18080.htm

Cappon, G., Vettoretti, M., Sparacino, G., \& Facchinetti, A. (2019). Continuous Glucose Monitoring Sensors for Diabetes Management: A Review of Technologies and Applications. Diabetes \& metabolism journal, 43(4), 383-397. https://doi.org/10.4093/dmj.2019.0121

Chan, J., \& Cheng-Lai, A. (2017). Inhaled Insulin: A Clinical and Historical Review. Cardiology in review, 25(3), 140-146. https://doi.org/10.1097/CRD.0000000000000143

de M Bandeira, S., da Fonseca, L. J., da S Guedes, G., Rabelo, L. A., Goulart, M. O., \& Vasconcelos, S. M. (2013). Oxidative stress as an underlying contributor in the development of chronic complications in diabetes mellitus. International journal of molecular sciences, 14(2), 3265-3284. https://doi.org/10.3390/ijms14023265

Diabetes: pesquisa avalia os fatores associados à qualidade de vida. (2019). Fundação Oswaldo Cruz. Acesso em: 22 de setembro de 2021. https://portal.fiocruz.br/noticia/diabetes-pesquisa-avalia-os-fatores-associados-qualidade-de-vida

Diretrizes Sociedade Brasileira de Diabetes. (2019). Saúde Bahia. Acesso em: 15 de novembro de 2021. http://www.saude.ba.gov.br/wpcontent/uploads/2020/02/Diretrizes-Sociedade-Brasileira-de-Diabetes-2019-2020.pdf

Gil, A. C. (2022). Como elaborar projetos de pesquisa. Atlas.

Hegele, R. A., \& Maltman, G. M. (2020). Insulin's centenary: the birth of an idea. The lancet. Diabetes \& endocrinology, 8(12), 971-977. https://doi.org/10.1016/S2213-8587(20)30337-5

Insulin Basics. (n.d.). American Diabetes Association. https://www.diabetes.org/healthy-living/medication-treatments/insulin-other-injectables/insulin-basics

International Diabetes Federation. (2019). IDF Diabetes Atlas, 9th ed. https://www.diabetesatlas.org

International Working Group On The Diabetic Foot (IWGDF). (n.d) International Consensus on the Diabetic Foot and Practical Guidelines. Acesso em: 15 de outubro de 2021. http://iwgdf.org/consensus

Kim, E. S., \& Plosker, G. L. (2015). AFREZZA® (insulin human) Inhalation Powder: A Review in Diabetes Mellitus. Drugs, 75(14), 1679-1686. https://doi.org/10.1007/s40265-015-0472-0

Klafke, A., Duncan, B. B., Rosa, R. S., Moura, L., Malta, D. C., \& Schmidt, M. I. (2014). Mortalidade por complicações agudas do diabetes melito no Brasil, 2006-2010. Epidemiologia e Serviços de Saúde. 23(3), 455-462. https://doi.org/10.5123/S1679-49742014000300008 
Kravarusic, J., \& Aleppo, G. (2020). Diabetes Technology Use in Adults with Type 1 and Type 2 Diabetes. Endocrinology and metabolism clinics of North America, 49(1), 37-55. https://doi.org/10.1016/j.ecl.2019.10.006

Malik, F. S., \& Taplin, C. E. (2014). Insulin therapy in children and adolescents with type 1 diabetes. Paediatric drugs, 16(2), 141-150. https://doi.org/10.1007/s40272-014-0064-6

Marques, R. d. M. B., Fornés, N. S., \& Stringhini, M. L. F. (2011). Fatores socioeconômicos, demográficos, nutricionais e de atividade física no controle glicêmico de adolescentes portadores de diabetes melito tipo 1. Arquivos Brasileiros de Endocrinologia \& Metabologia, 55(3), 194-202. https://doi.org/10.1590/s0004-27302011000300004

Molitch, M. E., DeFronzo, R. A., Franz, M. J., Keane, W. F., Mogensen, C. E., Parving, H. H., Steffes, M. W., \& American Diabetes Association (2004). Nephropathy in diabetes. Diabetes care, 27(1), S79-S83. https://doi.org/10.2337/diacare.27.2007.s79

Nascimento, O. J. M. d., Pupe, C. C. B., \& Cavalcanti, E. B. U. (2016). Diabetic neuropathy. Revista Dor, 17. https://doi.org/10.5935/1806-0013.20160047

Nimri, R., Nir, J., \& Phillip, M. (2020). Insulin Pump Therapy. American journal of therapeutics, 27(1), e30-e41. https://doi.org/10.1097/MJT.0000000000001097

Números do Diabetes no Brasil. (2021). Sociedade Brasileira de Endocrinologia e Metabologia. Acesso em 15 de novembro de 2021. https://www.endocrino.org.br/numeros-do-diabetes-no-brasil/

O que é insulina e tipos de insulina. (2021). BD. Acesso em: 13 de outubro de 2021. https://www.bd.com/pt-br/our-products/diabetes-care/diabetes-learningcenter/insulin-treatment/what-is-insulin-and-insulin-types

Owens D. R. (2011). Insulin preparations with prolonged effect. Diabetes technology \& therapeutics, 13 Suppl 1, S5-S14. https://doi.org/10.1089/dia.2011.0068

Premarket Notification. (2021). Food \& Drug Administration (FDA). https://www.accessdata.fda.gov/scripts/cdrh/cfdocs/cfPMN/pmn.cfm? start_search=1\&produc tcode=LZG\&knumber=\&applicant=INSULET\%20CORPORATION

Premarket Approval (PMA). (2021). Food \& Drug Administration (FDA). https://www.accessdata.fda.gov/scripts/cdrh/cfdocs/cfpma/pma.cfm?ID=P160017

Premarket Approval (PMA). (2021). Food \& $\quad$ Drug Administration $\quad$ (FDA). https://www.accessdata.fda.gov/scripts/cd rh/cfdocs/cfpma/pma.cfm?id=P140015S020

Pronin, T. (2019). Custo faz jovens racionarem insulina nos EUA; por que remédio lá é caro? https://www.uol.com.br/vivabem/noticias/redacao/2019/09/27/custo-faz-jovens-racionarem-insulina-nos-eua-por-que-remedio-e-tao-caro.htm

Retnakaran, R., \& Zinman, B. (2021). The ongoing evolution of basal insulin therapy over 100 years and its promise for the future. Diabetes, obesity \& metabolism, 10.1111/dom.14552. Advance online publication. https://doi.org/10.1111/dom.14552

Ross, L. J., \& Neville, K. A. (2019). Continuous subcutaneous insulin infusion versus multiple daily injections for type 1 diabetes. Journal of pediatrics and child health, 55(6), 718-722. https://doi.org/10.1111/jpc. 14480

Saleem, F., \& Sharma, A. (2021). NPH Insulin. In StatPearls. StatPearls Publishing. https://www.ncbi.nlm.nih.gov/books/NBK549860/

Scheiner Gary, MS, CDE. (2020). Think Like a Pancreas: A Practical Guide to Managing Diabetes with Insulin. Hachette Go (3a ed.).

Singh, V. P., Bali, A., Singh, N., \& Jaggi, A. S. (2014). Advanced glycation end products and diabetic complications. The Korean journal of physiology \& pharmacology: official journal of the Korean Physiological Society and the Korean Society of Pharmacology, 18(1), 1-14. https://doi.org/10.4196/kjpp.2014.18.1.1

Site do Accu-chek. Accu-chek. https://www.accu-chek.com.br/br/ids/combo/

Tarr, J. M., Kaul, K., Wolanska, K., Kohner, E. M., \& Chibber, R. (2012). Retinopathy in diabetes. In Advances in experimental medicine and biology (pp. 88106). Springer New York. https://doi.org/10.1007/978-1-4614-5441-0_10

Tibaldi J. M. (2014). Evolution of insulin: from human to analog. The American journal of medicine, 127(10 Suppl), S25-S38. https://doi.org/10.1016/j.amjmed.2014.07.005

Umpierrez, G. E., \& Klonoff, D. C. (2018). Diabetes Technology Update: Use of Insulin Pumps and Continuous Glucose Monitoring in the Hospital. Diabetes care, 41(8), 1579-1589. https://doi.org/10.2337/dci18-0002

van Belle, T. L., Coppieters, K. T., \& von Herrath, M. G. (2011). Type 1 diabetes: etiology, immunology, and therapeutic strategies. Physiological reviews, 91(1), 79-118. https://doi.org/10.1152/physrev.00003.2010

Wu, Y., Ding, Y., Tanaka, Y., \& Zhang, W. (2014). Risk factors contributing to type 2 diabetes and recent advances in the treatment and prevention. International journal of medical sciences, 11(11), 1185-1200. https://doi.org/10.7150/ijms.10001 